\title{
Persistence and regredience of intraspinal fluid collection determine symptom control in intracranial hypotension syndrome
}

\author{
Gereon Johannes Schnellbächer ${ }^{1}\left(\right.$ D $\cdot$ Michael Mull $^{2,3} \cdot$ Arno Reich $^{1}$ \\ Received: 1 February 2020 / Accepted: 18 July 2020 / Published online: 3 August 2020 \\ (C) The Author(s) 2020
}

\begin{abstract}
Background and purpose An intraspinal fluid collection (ISFC) can be observed on spinal MRI in cases of intracranial hypotension syndrome (IHS). The goal of this study was to analyze the possible persistence of ISFC after therapy and its correlation to clinical disease activity and secondary complications.

Materials and methods Twenty patients in our database of 57 patients, who were treated for IHS between 2009 and 2015, fulfilled the inclusion criteria of (a) diagnosed and treated IHS as well as (b) an ISFC in MRI imaging. Ten of these participated in our study. We performed follow-up visits, which included a history, a clinical examination, and a spinal MRI.

Results A MRI-confirmed ISFC was seen in six patients, five of which had symptoms attributable to chronic IHS. There were two cases of superficial siderosis. One patient had a persisting ISFC and was free of symptoms. Four patients did not have an ISFC and were free of symptoms (Fisher's exact test; $p<0.048$ ).

Conclusion There is statistically significant correlation between the persistence of an ISFC after IHS treatment and ongoing clinical symptoms. Resolved symptoms seem to correlate with absorbed extradural ISFC and hypothetically closed leakage site. ISFC as confirmed by MRI proofs to be a reliable follow-up marker for disease activity in chronic IHS that is possibly even superior to clinical examination.
\end{abstract}

Keywords Intracranial hypotension $\cdot$ Headache $\cdot$ Intraspinal fluid collection $\cdot$ Superficial siderosis

\section{Introduction}

Intracranial hypotension syndrome (IHS) is a consequence of cerebrospinal fluid (CSF) hypovolemia, caused by spontaneous or (micro-)traumatic CSF leakage into extra-arachnoidal

Michael Mull and Arno Reich contributed equally to this work.

Gereon Johannes Schnellbächer gschnellbaecher@ukaachen.de

Michael Mull

mmull@ukaachen.de

Arno Reich

areich@ukaachen.de

1 Department of Neurology, RWTH Aachen University, Pauwelsstrasse 30, D-52074 Aachen, Germany

2 Department of Diagnostic and Interventional Neuroradiology, RWTH Aachen University, Aachen, Germany

3 Department of Neuroradiology, RWTH Aachen University, Pauwelsstrasse 30, D-52074 Aachen, Germany and extradural spinal spaces [1]. The estimated annual incidence is 5 per 100,000 with a midlife peak and a female predominance [2]. The diagnostic criteria are (A) any headache fulfilling criteria $\mathrm{B}$ through $\mathrm{D}$ with (B) low CSF pressure $\left(<60 \mathrm{mmH}_{2} \mathrm{O}\right)$ and/or evidence of CSF leakage on imaging; (C) headache has developed in temporal relation to the low CSF pressure or CSF leakage or has led to its discovery (D) not better accounted for by another ICHD-3 diagnosis (Headache Classification Committee of the International Headache Society 2013). The overall spectrum of symptoms besides headaches is more diverse and commonly includes photophobia, dizziness, and tinnitus. Neurocognitive decline and brainstem ischemias have also been reported $[3,4]$.

In the case of chronic IHS, the headache may change from an initial postural character with severe headaches while standing into a milder and constant bilateral throbbing without clear orthostatic features [5]. This is important since it adds to the obscure nature of the disease and makes clinical observation more difficult. Although generally considered to be benign, especially chronic IHS may lead to secondary complications, such as cerebral sinus thrombosis and superficial 
siderosis (SS) [6], with potentially irreversible damage and in some cases even lethal consequences [7,8]. These long-term sequels further expand the spectrum of symptoms that can be associated with an underlying chronic IHS pathology.

A variety of possible causes for the development of IHS exist including iatrogenic after surgery $[9,10]$. In the past, a large proportion of IHS cases were considered to be idiopathic, since no clear etiology could be identified. In recent years, it has been suggested that microspurs and meningeal diverticula might explain many of those cases [11]. Independent of etiology spinal leakage leads to a common final path with CSF hypovolemia and extra-arachnoidal or extradural spinal fluid collection. First-line treatment is usually conservative, i.e., bedrest, head-down position, and administration of caffeine or theophylline, speculating on spontaneous closure of the leak over time. The next or alternative step is the application of repetitive blood patches without guidance. In cases of an identified leakage, CT-guided blood patches are performed $[12,13]$. Moreover, an operative closure by administration of fibrin glue or a suture can be pursued.

The increased application of MRI frequently identifies not only supratentorial pathologies associated with IHS like subdural hematoma or the enhancement of the meninges but also an intraspinal fluid collection (ISFC) [14]. This fluid is hypothesized to be the accumulated leaked CSF. The exact anatomic compartment, in which spinal fluid collects, is sometimes difficult to assess and may vary from case to case. Both subdural and extradural extravasations of fluids have been described before [5]. In this study, the more general term ISFC is used to avoid confusion. In cases of chronic IHS with ISFC, the development of a SS is thought to be a serious complication. Hence, failure in leakage closure and ISFC resorption may lead to irreversible structural lesions of the central nervous system. The efficacy of the blood patches or surgery was often correlated to the reversal of cerebral abnormalities $[15,16]$; however, more recently spinal alterations have become the focus of attention as well $[17,18]$. The aim of the following study was to clarify the association of clinical disease activity with regard to persistence or regression of ISFC in chronic IHS and to study long-term sequels with regard to the occurrence of a cerebral hemosiderosis. We postulated that spinal MRI is a necessary and effective tool to monitor disease activity.

\section{Methods}

\section{Patient selection}

Adult patients who were treated at our university hospital between 2009 and 2015 were screened for eligibility, namely, the diagnosis of IHS and the presence of ISFC before treatment (Fig. 3). We used a search algorithm with the term "intracranial hypotension syndrome" for all patients treated in our institution in the above-mentioned time period. This resulted in 153 possible cases. After studying of the records, the diagnosis of IHS was confirmed in 57 patients. They had been dismissed from hospital care after resolution or reduction of the initial symptoms. Twenty-seven patients had suffered from a significant orthostatic headache after lumbar puncture, which could be treated without complications. The other 30 cases were diagnosed with an IHS of varying etiologies, such as spontaneous, after orthopedic infiltration therapy or traumatic. Of those 20 patients demonstrated a clear ISFC on MR imaging, 11 were willing to participate in the study and a written informed consent was obtained and 9 patients did not consent to participation. The study was approved by the local Ethics Committee and is in accordance with the Declaration of Helsinki.

\section{Examination and imaging}

Since the occurrence of an ISFC had been described before [14], spinal MRI was routinely performed to screen for abnormalities. Additionally, postmyelographic CT imaging of the entire spine was obtained via lumbar puncture, and in most cases, the contrast medium was injected with the patient positioned in the CT table. Based on the area of interest, examination was started in a supine or prone position. In one patient, digital subtraction myelography was added, because CT myelography failed to show the CSF leak. The detailed techniques of $\mathrm{CT}$ myelography and digital subtraction myelography are described elsewhere [19-21]. Prospectively, we performed a follow-up visit consisting of a neurological examination with special focus on the history of headaches and signs of ongoing or newly acquired peripheral or central nerve symptoms. Symptoms were considered to be chronic, if they persisted for more than 3 months. Furthermore, a 3 Tesla MRI was used for the follow-up neuroradiological examination. Imaging protocol included sagittal imaging of the entire spinal column with T2-TSE, T1-TSE, T2* (MEDIC) sequences. The slice thickness was $3 \mathrm{~mm}$. In addition, the region of the initial leak was examined axially with T2-TRUFI, T1-TSE; T2-TSE, T2* (MEDIC) sequences with a slice thickness of $3 \mathrm{~mm}$.

\section{Statistical methods}

The data was transferred on a fourfold table. A two-tailed Fisher's exact test was used for statistical analysis. A $p$ value of $\leq 0.05$ was considered to be significant. Outcome variables were the existence or absence of ISFC in relation to symptoms typical for a chronic IHS. For the calculation, SPSS 23 was used. 


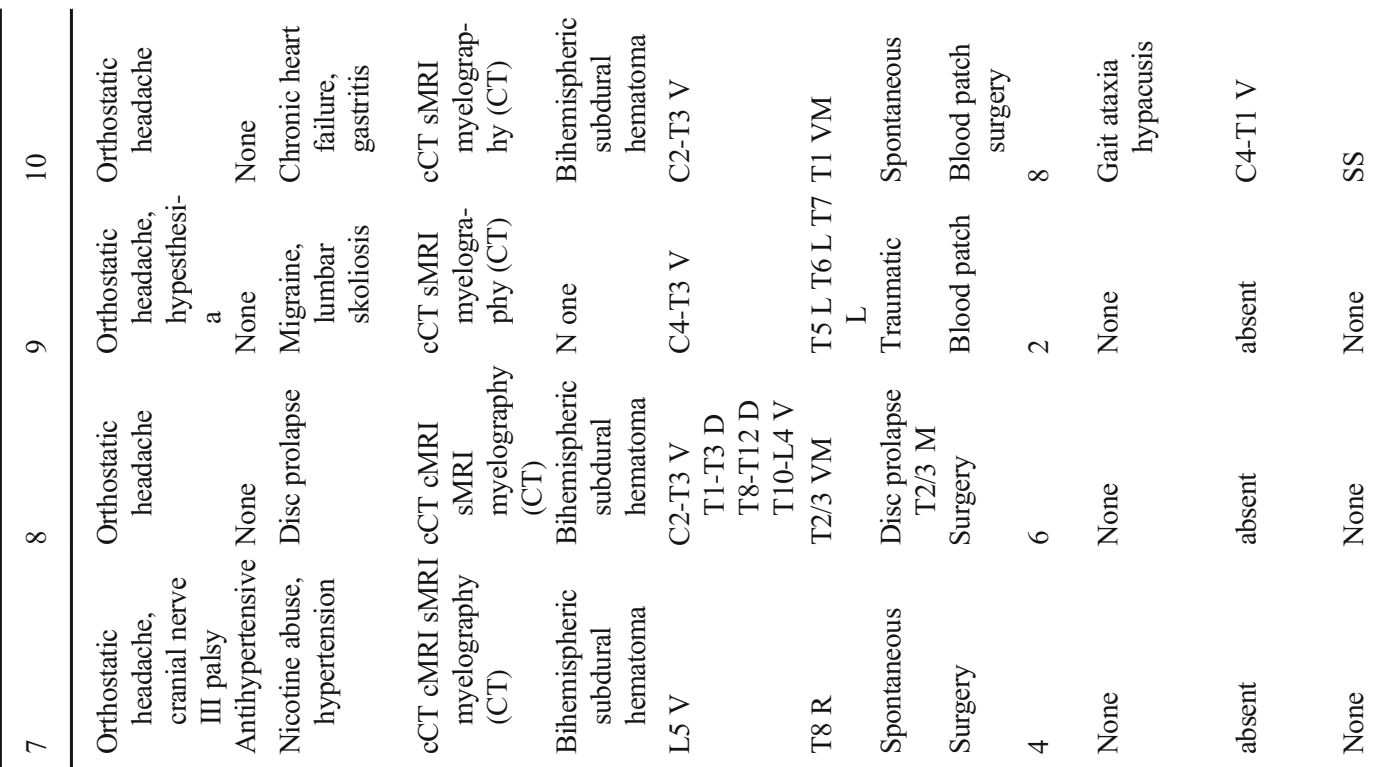

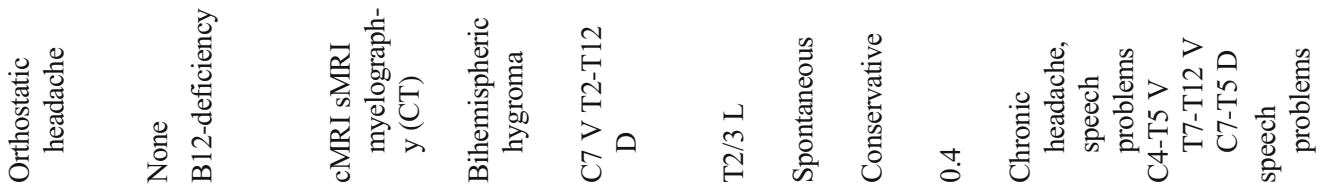

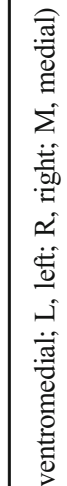

ナ

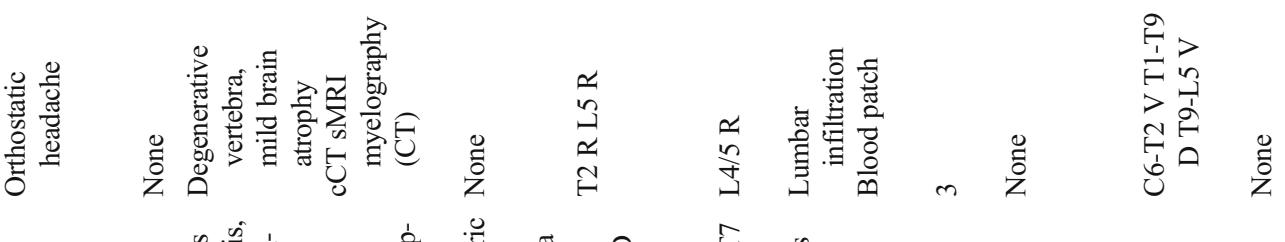

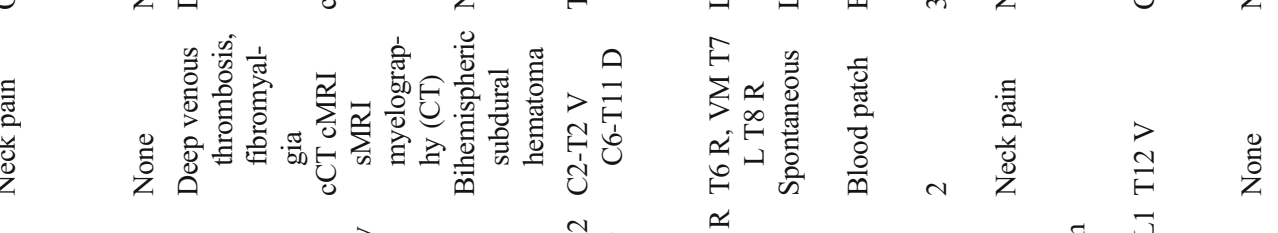

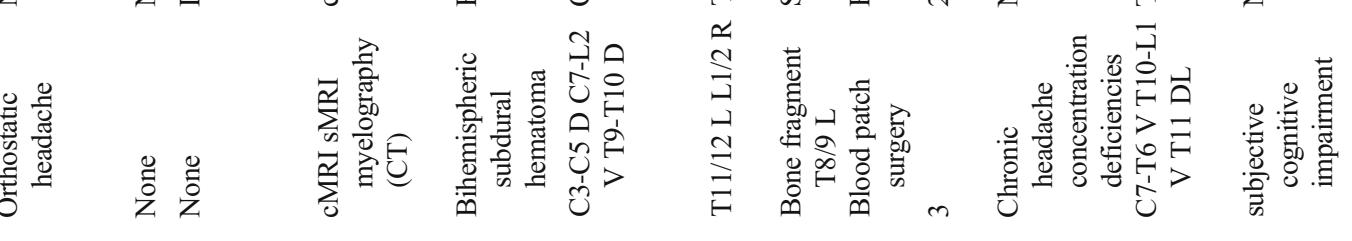

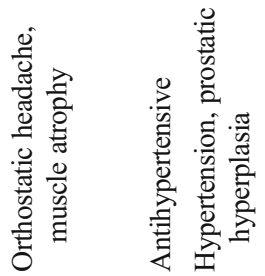

블.

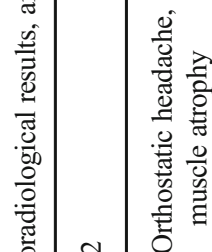

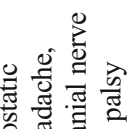

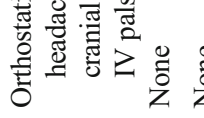

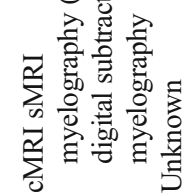

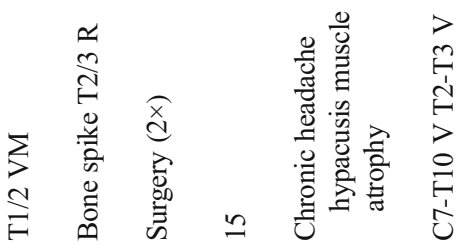

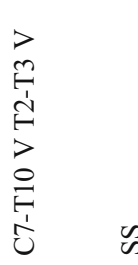

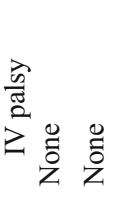

究

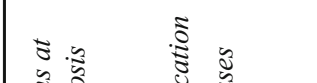

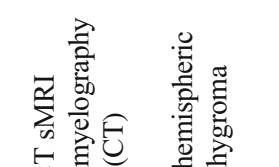

可矛 兽

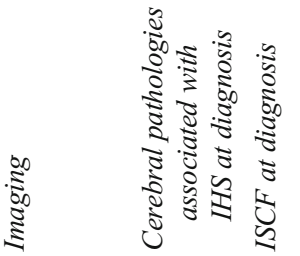

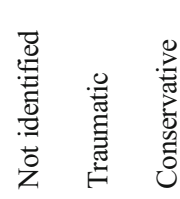

经

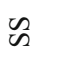

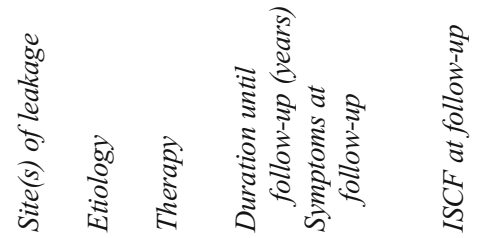

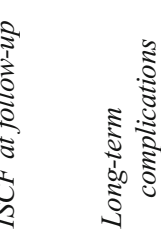

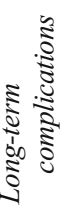


Table 2 Fourfold table correlating chronic IHS symptoms to the persistence of ISFC at the time of follow-up

$\begin{aligned} & \text { Chronic IHS } \\ & \text { symptoms }\end{aligned}$
No chronic IHS symptoms

symptoms

Intraspinal fluid 5

No intraspinal fluid 0

4

\section{Results}

Ten patients were examined and included in our data analysis. The eleventh patient underwent radiological follow-up examination, stated to be free of symptoms, but did not attend neurological follow-up examination. Since he did not comply with the study protocol, he had to be excluded. Of those participating in the study, four were female and six male (medium age 51.1 years). Median time from therapy to follow-up was 3 years (Q1 2 years, Q3 6 years).

\section{Clinical presentation}

All showed as initial symptom of orthostatic headaches with one exception (case no. 4), where neck pain was the leading symptom. In this case, an IHS was diagnosed due to the presence of bihemispheric hygromas, ISFC, and identification of a thoracic dural leak. Four patients had additional focal neurological deficits (Table 1).

At the time of follow-up examinations, IHS-related headache symptoms were completely reversible in six patients. One patient described both a postural and non-postural headache. Two patients suffered from a chronic headache that did not show an orthostatic character. One patient reported a reduction of the initial neck pain. A paresis of the abducens nerve was persistent in one case after otherwise successful treatment possibly as a sign of irreversible nerve damage. Two cases developed over the course of up to 13 years a SS with ataxia and hypacusis at the time of follow-up. Furthermore, two patients complained about problems with concentration and verbal deficiency, respectively (Table 1).

\section{Neuroradiological presentation}

Most of the leaks were found on the thoracic level $(n=8)$ at diagnosis. In one patient, the leak was located in the lumbar region. In one case, the site of the leakage could not be found. ISFC localization varied with four cases demonstrating extradural liquor ventrally, four cases both dorsally and ventrally, one case showed a right-sided ISFC, and one case only dorsally. Subdural hematomas or hygromas were present in seven cases.

At the time of follow-up examination ISFC could still be observed in six cases. Three of those had an ISFC ventral of the spinal cord and three demonstrated a fluid collection both ventrally and dorsally. From those cases still demonstrating an ISFC, in five several spinal levels were involved. In one case, ISFC could only be observed on level T12. Four patients demonstrated an increase of ISFC extent, while two patients showed a decrease. In four cases, the MRI demonstrated complete absorption the ISFC.

\section{Statistical analysis}

Of ten patients, four had neither any symptoms nor a residual fluid collection at the time of follow-up. In one case, there was an absence of IHS while ISFC was still detectable. Five patients showed persisting symptoms and extradural fluid collections. There was no patient with ongoing symptoms while lacking a spinal fluid collection. The results were summarized in a fourfold table (Table 2) with statistical significance of $p=$ 0.04 .

\section{Discussion}

Spinal imaging has become an essential part in the diagnosis and therapy of IHS [22]. Identification and localization of the CSF leak(s) may be difficult and influenced by the flow character in the leakage (low flow versus high flow). Different myelographic techniques (fast or ultra-fast CT myelography, digital subtraction myelography) were developed to overcome this problem $[19,20]$. Spinal MRI can demonstrate various secondary spinal manifestations of IHS like ISFCs, dilated veins, and dural enhancement [2]. Supratentorial changes, as, for example, subdural hygromas, that are usually included into follow-up examination to ascertain regression are increasingly complemented by spinal imaging as well [18]. Since repeated or chronic microbleeds in IHS cases with an associated ISFC can lead to complications like SS [23] with potentially irreversible neurological deficits, spinal imaging rightfully deserves our attention. It has been theorized that drag caused by intracranial hypotension leads to an engorgement of intradural veins causing the subsequent microbleeds [24] and accumulation of hemosiderin on the brain surface and its subpial layers $[25,26]$ resulting in a cytotoxic effect through free iron and hydroxyl radicals [27]. Etiology of SS may vary with traumas, arteriovenous malformations, and tumors being among others [6]. However, in about half of the patients, no etiology is identified [28]. It is in these cases one has to be aware of the possibility of an underlying chronic IHS, which may be identified by the diagnosis of an ISFC. In cases \#2 (Fig. 2) and \#10, the leak persisted despite of the regression of orthostatic headache and led over a period of 15 and 8 years, respectively, to the development of a SS with debilitating symptoms like among others gait ataxia and hypacusis. Case \#2 demonstrated SS of significant extent affecting frontal, 
Fig. 1 Preoperative sagittal and axial (inset) T2-weighted MR images of the cervicothoracic and lumbar regions $(\mathbf{a}, \mathbf{b})$ reveal extensive anterior intraspinal fluid collection from $\mathrm{C} 5 / 6$ to $\mathrm{T} 11$ (arrows). Note hypointense formation of the distal thecal sac due to hemosiderin deposits at $\mathrm{S} 1 / 2$ (arrowhead). Postoperative sagittal T2-weighted MR images (c) 13 months after repair of the T1/2 dural tear shows persisting resolution of the fluid collection. Gradient echo MR images (d, e) clearly detect hemosiderin depositions along and around the cord associated with cord atrophy, as well as along the cerebellar folia, around midbrain and pons (arrows)
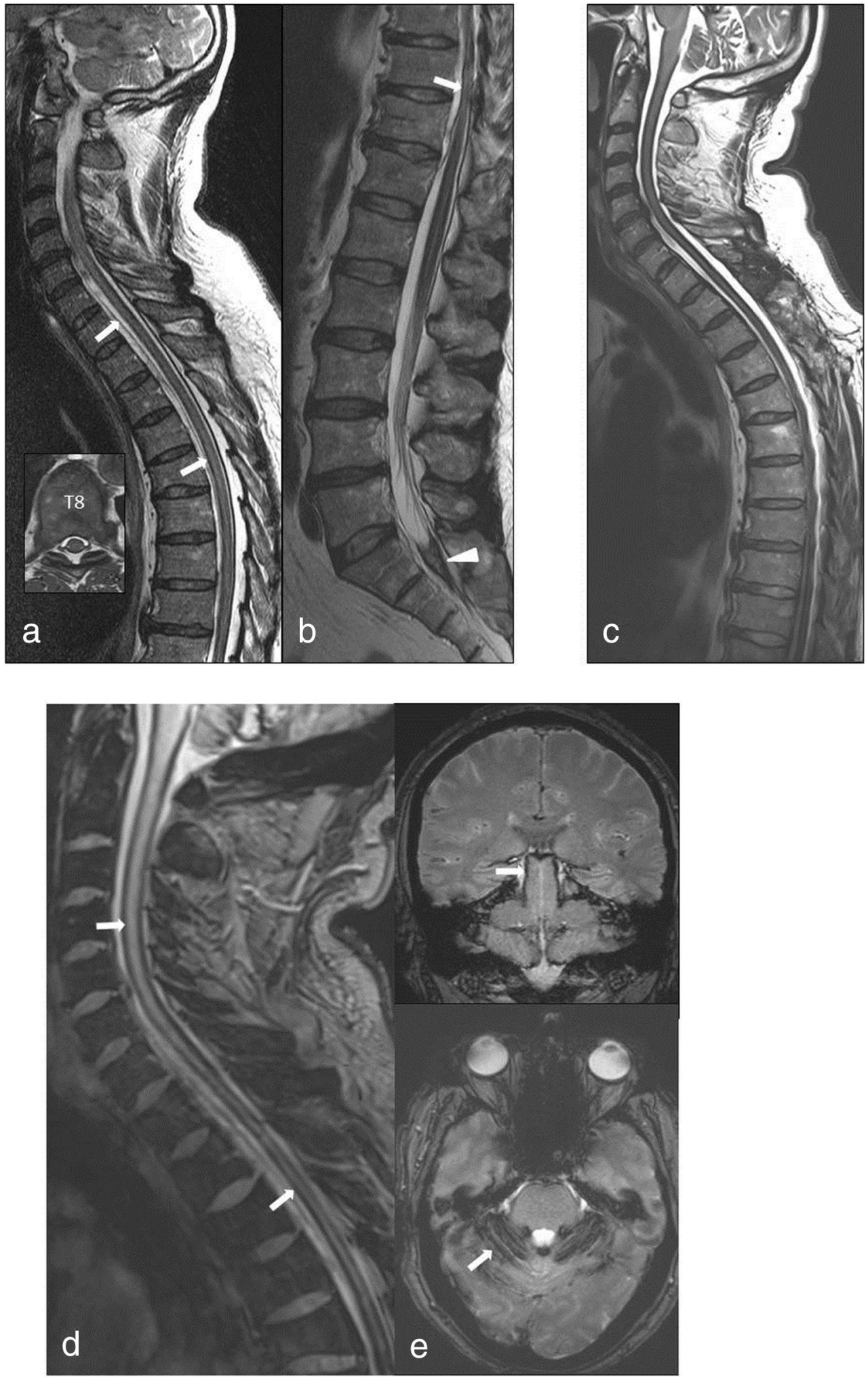

sulcal, occipital, and intrathecal structures possibly due to the long time course disease progression was not stopped.

Not every leak seems to be accompanied by classical symptoms of IHS. Case \#5 (Fig. 3), which originally demonstrated a recurrent leak at level L4/5 following facet infiltration, showed an extensive intraspinal fluid collection at the time of follow-up. However, the initial symptoms of IHS had ceased, and the patient presented himself free of 
Fig. 2 Initial sagittal T2-weighted MR images of the cervicothoracic and lumbar regions (a) demonstrate extensive anterior and posterior intraspinal fluid collections from $\mathrm{C} 1$ down to $\mathrm{S} 1$ (arrows). Coronal and axial reformatted CT myelography (b) reveals rightsided CSF leaks at the lumbar roots L2, L3, and L4 (arrowheads). Resolution of the intraspinal fluid collections is documented in sagittal T2weighted MR images 1 month after a single lumbar CT-guided blood patch application (c). De novo formation of the intraspinal fluid collection (arrows) is observed without clinical symptoms in the late follow-up MR 3 years later (d)
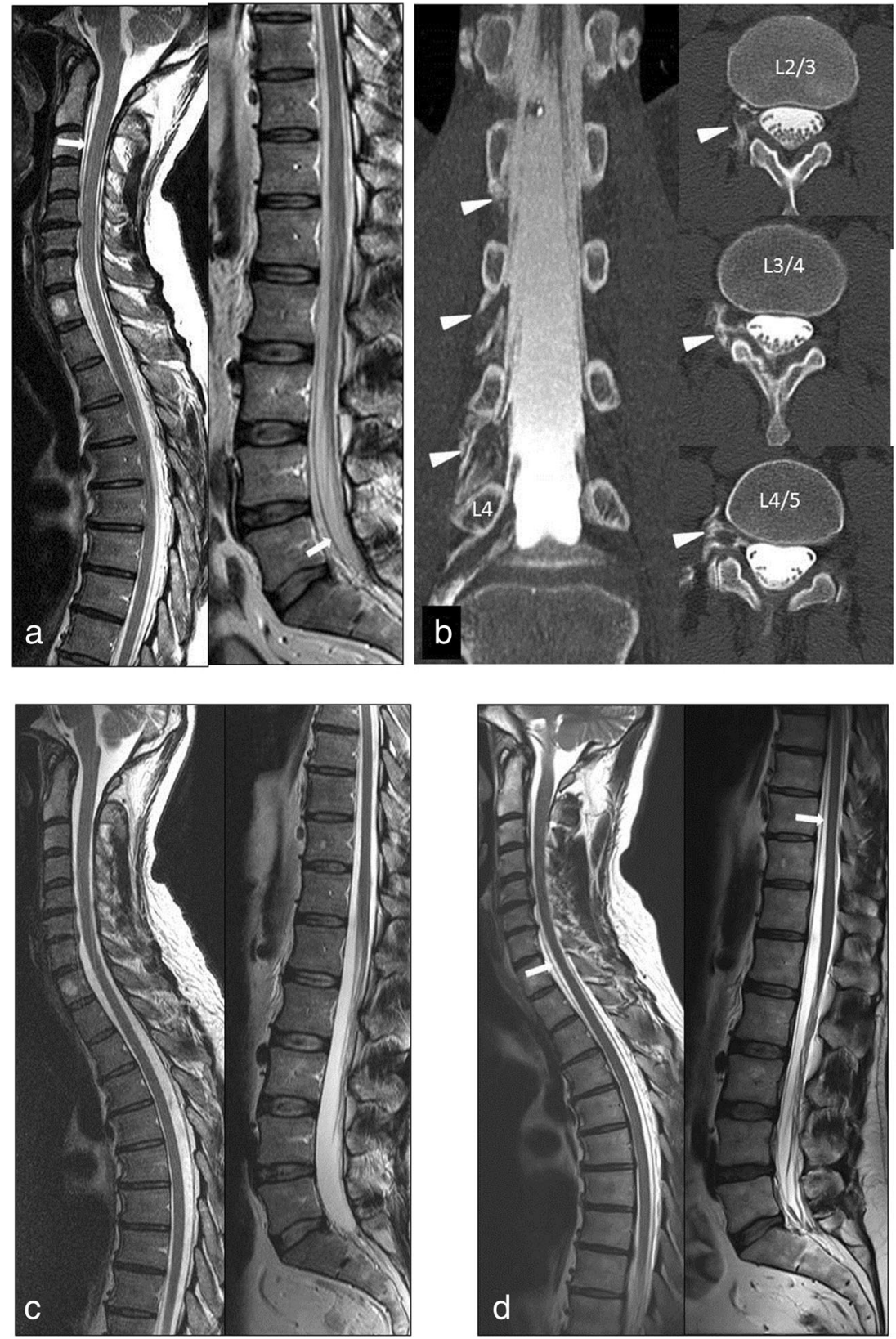

complaints. A possible explanation is the development of a new equilibrium between the different spinal compartments and the surrounding connective tissue preventing further spreading while the leak itself remains unclosed. According to the Kelly-Monroe doctrine, the space within the central nervous system is limited. If one of the containing elements therein - blood, liquor, and brain parenchyma - increases in volume, the others must decrease. Through an extradural extension of liquor, this above-mentioned principle may be compensated and clinical symptoms may not be manifested. This may add to the variable and inconclusive clinical picture of chronic IHS and emphasizes the need for neuroradiological control examinations (Fig. 3).
To our knowledge, this is the first study analyzing a longterm follow-up of IHS. In our cohort of patients, the median time interval from therapy to follow-up exam was 3 years. While this might be too short for reliably detecting SS, it can identify a recurrent or not regressing ISFC as described in case \#5. It is of interest to note that both cases with SS had a particularly long time interval from therapy to follow-up. This indicates that complications of chronic IHS can manifest themselves even more than a decade after diagnosis. Figure 4 proposes a decision tree to be used in case of IHS. If an ISFC accompanies the diagnosis of an IHS, spinal follow-up examination is always recommended regardless of symptom control or regression. In our diagram, we propose an examination 
Fig. 3 Flow-chart showing number of patients included in study

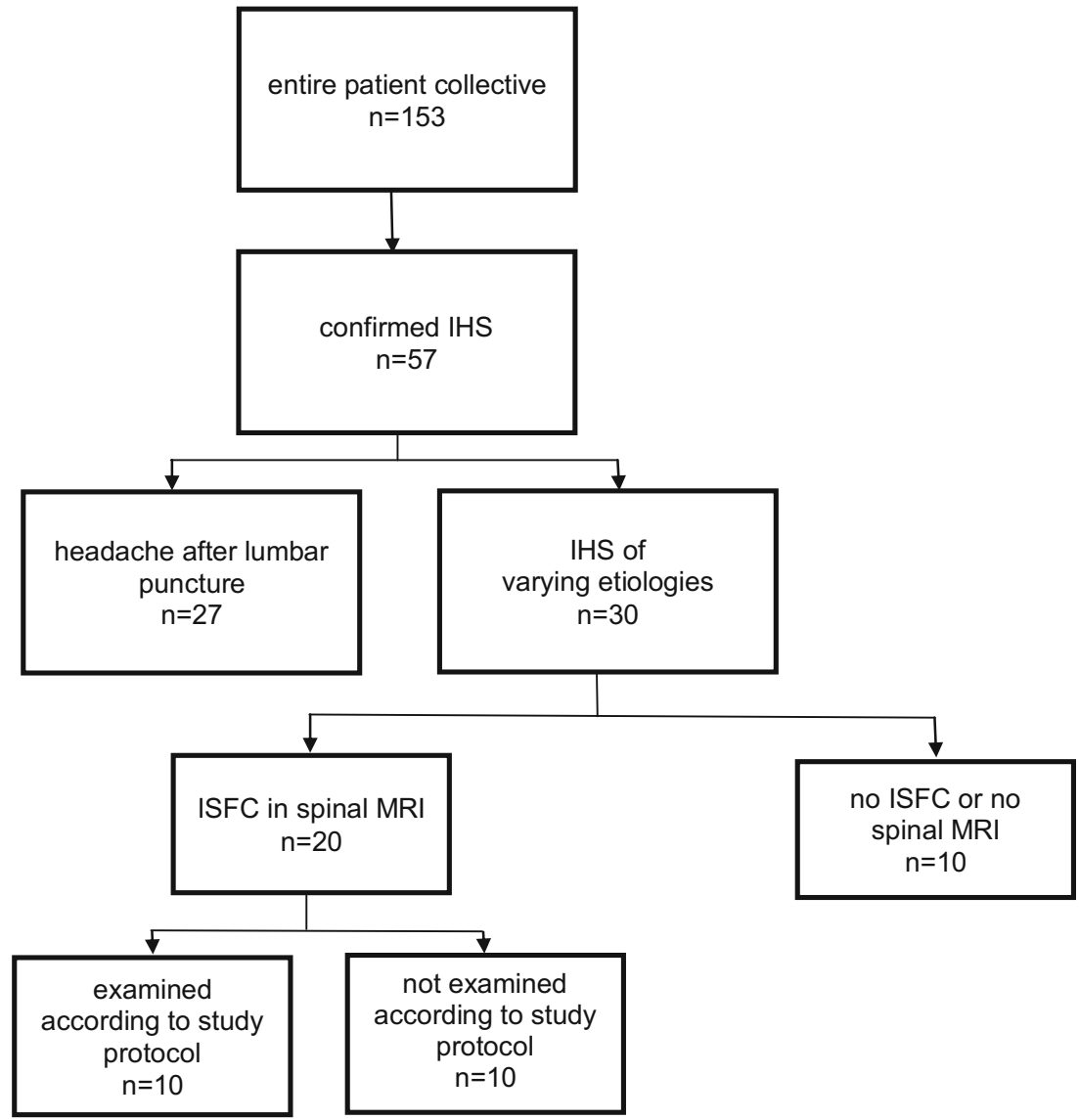

Fig. 4 Decision tree for diagnostic evaluation

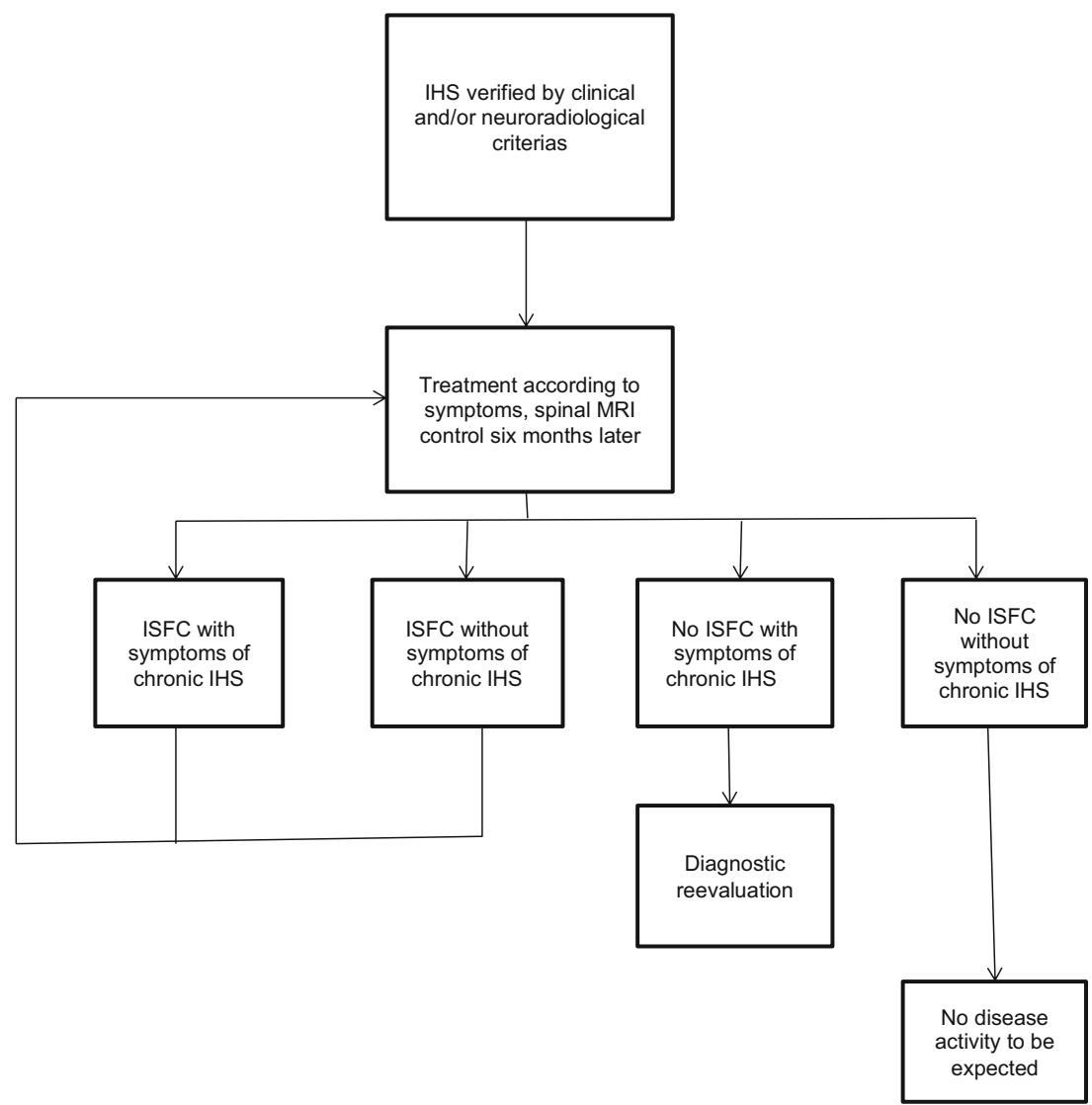


interval after therapy of 6 months to ascertain reduction of the ISFC. When no neuroradiological signs are present, the opaque nature of the clinical appearance of chronic IHS can make diagnosis difficult. In this case, a diagnostic reevaluation should take place in order not to miss possible treatable other causes for the complaints. In patients free of ISFC and without symptoms, no disease activity is to be expected.

The study was not designed to determine the incidence of an ISFC in patients with IHS. However, our retrospective analysis of patients with chronic IHS in our institution suggests that an ISFC can be found in a significant percentage of cases.

The protocol of spinal examinations should encompass T1 and T2 spin echo sequences for demonstration of intraspinal fluid collections. While the T2 spin echo sequence is effective in showing the dural sac, the $\mathrm{T} 1$ sequence is sensitive to the extradural fluid collection itself and useful to differentiate from epidural fat. A T2* or SWI sequence should be chosen to detect more sensitively possible cases of SS [29]. Another GRE sequence T2-Trufi can reduce the impact of field inhomogeneities caused by motion for an improved visualization of CSF.

Limitations of our study were its single-center design and the varying post-therapeutic follow-up intervals. The low number of patients recruited is problematic, although results were statistically highly significant. Further investigations could help improve both patient care and our understanding of a still insufficiently comprehended disease. Our data shows that persistence and dynamic of intraspinal fluid collections were predictive of chronic IHS-associated symptoms. All patients with a resolved fluid collection were free of symptoms on follow-up, while apart from one exception (case \#5), all patients with persistent fluid collections suffered from ongoing complaints attributable to IHS disease. It can therefore be postulated that in case of successful closure of a leak, the intraspinal fluid is gradually absorbed and that the hypothesis of that spinal MRI is a valid tool for monitoring disease activity in IHS is true. It even seems more reliable than clinical examination since disease activity is often opaque and unspecific. It should routinely be used not only in the diagnosis of IHS but also in follow-up examinations. This is recommended not only for patients with ongoing chronic IHS symptoms but also for asymptomatic patients in order not to miss treatment opportunities and to prevent irreversible long term sequelae such as SS.

Acknowledgements Open Access funding provided by Projekt DEAL.

\section{Compliance with ethical standards}

Conflict of interest The authors declare no conflict of interest.

Ethical approval All procedures performed in studies involving human participants were in accordance with the ethical standards of the institutional research committee and with the 1964 Helsinki declaration and its later amendments or comparable ethical standards.

Consent to participate Informed consent was obtained from all individual participants included in the study.

Consent to publish The authors affirm that human research participants provided informed consent for publication of the images in Figs. 1 and 2.

Open Access This article is licensed under a Creative Commons Attribution 4.0 International License, which permits use, sharing, adaptation, distribution and reproduction in any medium or format, as long as you give appropriate credit to the original author(s) and the source, provide a link to the Creative Commons licence, and indicate if changes were made. The images or other third party material in this article are included in the article's Creative Commons licence, unless indicated otherwise in a credit line to the material. If material is not included in the article's Creative Commons licence and your intended use is not permitted by statutory regulation or exceeds the permitted use, you will need to obtain permission directly from the copyright holder. To view a copy of this licence, visit http://creativecommons.org/licenses/by/4.0/.

\section{References}

1. Paldino M, Maldiner AY, Tenner MS (2003) Intracranial hypotension syndrome: a comprehensive review. Neurosurg Focus 15: ECP2

2. Schievink WI (2006) Spontaneous spinal cerebrospinal fluid leaks and intracranial hypotension. JAMA 295:2286-2296

3. Amemiya S, Takahashi K, Mima T, Yoshioka N, Miki S, Ohtomo K (2016) Reversible alterations of the neuronal activity in spontaneous intracranial hypotension. Cephalalgia 36:162-171

4. Matosevic B, Prieschl M, Luef G, Knoflach M, Schmidauer C, Willeit J, Lackner P (2016) Recurrent brainstem infarction caused by spontaneous intracranial hypotension. Cephalalgia 36:812-813

5. Mokri B (2013) Spontaneous low pressure, low CSF volume headaches: spontaneous CSF leaks. Headache 53:1034-1053

6. Kumar N, Cohen-Gadol AA, Wright RA, Miller GM, Piepgras DG, Ahlskog JE (2006) Superficial siderosis. Neurology 66:1144-1152

7. Schievink WI, Wasserstein P, Maya MM (2016) Intraspinal hemorrhage in spontaneous intracranial hypotension: link to superficial siderosis? Report of 2 cases. J Neurosurg Spine 24:454-456

8. Sass C, Kosinsiki C, Schmidt P et al (2013) Intrathecal saline infusion: an emergency procedure in a patient with spontaneous intracranial hypotension. Neurocrit Care 19:116-118

9. Jankowitz BT, Atteberry DS, Gerszten PC et al (2009) Effect of fibrin glue on the prevention of persistent cerebral spinal fluid leakage after incidental durotomy during lumbar spinal surgery. Eur Spine J 18:169-174

10. Inamasu J, Guiot BH (2006) Intracranial hypotension with spinal pathology. Spine J 6:591-599

11. Beck J, Ulrich CT, Fung C, Fichtner J, Seidel K, Fiechter M, Hsieh K, Murek M, Bervini D, Meier N, Mono ML, Mordasini P, Hewer E, Z'Graggen WJ, Gralla J, Raabe A (2016) Diskogenic microspurs as a major cause of intractable spontaneous intracranial hypotension. Neurology 87:1220-1226

12. Berroir S, Loisel B, Ducros A, Boukobza M, Tzourio C, Valade D, Bousser MG (2004) Early epidural blood patch in spontaneous intracranial hypotension. Neurology 63:1950-1951

13. Griauzde J, Gemmete JJ, Chaudhary N, Wilson TJ, Pandey AS (2014) Large-volume blood patch to multiple sites in the epidural 
space through a single-catheter access site for treatment of spontaneous intracranial hypotension. Am J Neuroradiol 35:1841-1849

14. Rabin BM, Roychowdhury S, Meyer JR, Cohen BA, LaPat K, Russell EJ (1998) Spontaneous intracranial hypotension: spinal MR findings. Am J Neuroradiol 19:1034-1039

15. Huang CW, Tsai YF, Hsiao CY (2013) Different MRI signs in predicting the treatment efficacy of epidural blood patch in spontaneous intracranial hypotension: a case report. Iran J Radiol 10:7274

16. Schievink WI, Maya MM, Louy C et al (2005) Cranial MRI predicts outcome of spontaneous intracranial hypotension. Neurology 64:1282-1284

17. Wu JW, Hseu SS, Fuh JL, Lirng JF, Wang YF, Chen WT, Chen SP, Wang SJ (2017) Factors predicting response to the first epidural blood patch in spontaneous intracranial hypotension. Brain 140: 344-352

18. Wu JW, Wang YF, Fuh JL, Lirng JF, Chen SP, Hseu SS, Wang SJ (2018) Correlations among brain and spinal MRI findings in spontaneous intracranial hypotension. Cephalalgia 38:1998-2005

19. Hoxworth JM, Patel AC, Bosch EP, Nelson KD (2009) Localization of a rapid CSF leak with digital subtraction Myelography. Am J Neuroradiol 30:516-519

20. Thielen KR, Sillery JC, Morris JM, Hoxworth JM, Diehn FE, Wald JT, Rosebrock RE, Yu L, Luetmer PH (2015) Ultrafast dynamic computed tomography myelography for the precise identification of high-flow cerebrospinal fluid leaks caused by spiculated spinal osteophytes. J Neurosurg Spine 22:324-331. https://doi.org/10. 3171/2014.10.SPINE14209

21. Pomerantz SR (2016) Myelography: modern technique and indications. Handb Clin Neurol 135:193-208
22. Albes G, Weng H, Horvath D et al (2012) Detection and treatment of spinal CSF leaks in idiopathic intracranial hypotension. Neuroradiology 54:367-373

23. Kumar N, Miller GM, Piepgras DG, Mokri B (2010) A unifying hypothesis for a patient with superficial siderosis, low pressure headache, intraspinal cyst, back pain, and prominent vascularity. $\mathrm{J}$ Neurosurg 113:97-101

24. Sahin S, Agilkaya S, Karsidag S (2006) Superficial siderosis of the central nervous system: an unusual cause for headache and hearing loss. Neurol Asia 11:145-149

25. Gao JG, Zhou CK, Liu JY (2015) Superficial siderosis of the central nervous system: a case report. Exp Ther Med 9:1379-1382

26. Maurizi CP (1996) Superficial siderosis of the brain: roles for cerebrospinal fluid circulation, iron and the hydroxyl radical. Med Hypotheses 47:261-264

27. Kellermier H, Wang G, Wiley C (1996) Iron localization in superficial siderosis of the central nervous system. Neuropathology 29: 187-195

28. Calvo M, de Miquel C, Pinel A et al (2014) Diffuse superficial siderosis of the central nervous system: four case reports and review of the literature. Rev Neurol 16:354-358

29. Zimny A, Neska-Matuszewska M, Bladowska J et al (2015) Intracranial lesions with low signal intensity on T2-weighted MR images - review of pathologies. Pol J Radiol 25:40-50

Publisher's note Springer Nature remains neutral with regard to jurisdictional claims in published maps and institutional affiliations. 